\title{
West Nile virus and the central nervous system Kenneth L Tyler
}

Address: Departments of Neurology, Medicine, and Microbiology University of Colorado at Denver Health Sciences Center, Denver, CO 80262 USA, and Neurology Service Denver VA Medical Center, Denver, CO 80220 USA

Email: Kenneth L Tyler - ken.tyler@uchsc.edu

from Infectious diseases of the nervous system: pathogenesis and worldwide impact

Paris, France. 10-13 September 2008

Published: 23 September 2008

BMC Proceedings 2008, 2(Suppl I):S42

This abstract is available from: http://www.biomedcentral.com/I753-656I/2/SI/S42

(c) 2008 Tyler; licensee BioMed Central Ltd.

Following its emergence in the Western Hemisphere in 1999, West Nile virus (WNV) has become endemic and is now the most important cause of arboviral meningoencephalitis in the United States. Approximately 1\% of infected individuals develop neuroinvasive disease which can take the form of encephalitis, meningitis, or acute flaccid paralysis (poliomyelitis-like illness). The current epidemiology and clinical features of the different forms of WNV neuroinvasive disease will be discussed. In distinction to many other forms of viral meningoencephalitis, WNV disease is frequently associated with a CSF neutrophil rather than lymphocyte predominant CSF pleocytosis. The CSF characteristics of WNV disease will be reviewed. Neuroimaging is less frequently abnormal in WNV encephalitis than in some other types of acute encephalitis (e.g. herpes simplex encephalitis). The basic neuroimaging features of WNV encephalitis will be reviewed including the predilection for abnormalities involving the basal ganglia, thalamus and upper brainstem. Although seizures are unusual in WNV encephalitis, it has been suggested that anteriorly predominant slowing on EEG may be a clue to the diagnosis. EMG/NCV abnormalities are characteristic of WNV AFP and can include absence or decrease in Compound Motor Action Potentials (CMAPs) with preserved Sensory Nerve Action Potentials (SNAPs) as well as the presence of EMG signs of denervation. Serology remains the mainstay of diagnosis, with detection of WNV specific IgM in CSF being generally diagnostic of WNV neurological disease. The kinetics and application of serology and other diagnostic tests including CSF PCR will be discussed. There is currently no known specific therapy for WNV infection, however a variety of clinical trials involving interferon-alpha, intravenous immunoglobulin (IVIG) with high titer WNV antibody (e.g. Omr-IgG-am), and humanized monoclonal antibody against WNV envelope protein domains have been initiated and will be reviewed. Several WNV vaccines have now been licensed for veterinary use and the current status of human vaccine development and trials will be reviewed. Although there is currently no evidence that WNV produces chronic, persistent or recurrent disease in immunocompetent humans, some studies of WNV infection have suggested that a significant number of individuals may have long-term sequelae following acute infection including possible cognitive impairment, alterations in mood, fatigue, weakness and pain. 